\title{
Telomere elongation is under the control of the RNAi-based mechanism in the Drosophila germline
}

\author{
Mikhail Savitsky, ${ }^{1,4}$ Dmitry Kwon, ${ }^{2,3}$ Pavel Georgiev, ${ }^{1}$ Alla Kalmykova, ${ }^{2,5}$ and Vladimir Gvozdev ${ }^{2,6}$ \\ ${ }^{1}$ Department of the Control of Genetic Processes, Institute of Gene Biology, Russian Academy of Sciences, Moscow 119334, \\ Russia; ${ }^{2}$ Department of Molecular Genetics of Cell, Institute of Molecular Genetics, Russian Academy of Sciences, Moscow \\ 123182, Russia; ${ }^{3}$ Department of Molecular Biology, Moscow State University, Moscow 119899, Russia; ${ }^{4}$ Centre for Medical \\ Studies of Oslo University, Moscow 199334, Russia
}

\begin{abstract}
Telomeres in Drosophila are maintained by transposition of specialized telomeric retroelements $\mathrm{HeT}$ - $\mathrm{A}$, TAHRE, and TART instead of the short DNA repeats generated by telomerase in other eukaryotes. Here we implicate the RNA interference machinery in the control of Drosophila telomere length in ovaries. The abundance of telomeric retroelement transcripts is up-regulated owing to mutations in the spn-E and $a u b$ genes, encoding a putative RNA helicase and protein of the Argonaute family, respectively, which are related to the RNA interference (RNAi) machinery. These mutations cause an increase in the frequency of telomeric element retrotransposition to a broken chromosome end. spn-E mutations eliminate $H e T-A$ and TART short RNAs in ovaries, suggesting an RNAi-based mechanism in the control of telomere maintenance in the Drosophila germline. Enhanced frequency of TART, but not $\mathrm{HeT}-\mathrm{A}$, attachments in individuals carrying one dose of mutant spn-E or aub alleles suggests that TART is a primary target of the RNAi machinery. At the same time, we detected enhanced $\mathrm{HeT}$-A attachments to broken chromosome ends in oocytes from homozygous spn-E mutants. Double-stranded RNA (dsRNA)-mediated control of telomeric retroelement transposition may occur at premeiotic stages, resulting in the maintenance of appropriate telomere length in gamete precursors.
\end{abstract}

[Keywords: Telomere; RNAi; retrotransposon; HeT-A; TART; germline]

Supplemental material is available at http://www.genesdev.org.

Received October 13, 2005; revised version accepted December 1, 2005.

The problems of end-under-replication and stability of linear chromosomes are resolved by telomeres. The lengthening of terminal regions of linear eukaryotic chromosomes is often provided by RNA-templated addition of repeated DNA by reverse transcriptase enzyme, telomerase (Bryan and Cech 1999; Chan and Blackburn 2002). In most eukaryotes, telomeric DNA is maintained by the action of telomerase, which is responsible for the synthesis of short 6-8-nucleotide (nt) arrays using an RNA component as a template (Greider and Blackburn 1985). In contrast, telomeres of Drosophila are maintained as a result of retrotranspositions of specialized telomeric non-long-terminal repeat (LTR) HeT-A, TAHRE, and TART retrotranspositions (Biessmann et al. 1992b; Levis et al. 1993; for review, see Pardue and DeBaryshe 2003; Abad et al. 2004b). Retrotransposons are also found

Corresponding authors.

${ }^{5}$ E-MAIL allakalm@img.ras.ru; FAX 7-095-1960221.

${ }^{6}$ E-MAIL gvozdev@img.ras.ru; FAX 7-095-1960221.

Article and publication are at http://www.genesdev.org/cgi/doi/10.1101/ gad.370206. in telomeric regions of such diverse organisms as Bombix mori (Okazaki et al. 1995; Takahashi et al. 1997), Chlorella (Higashiyama et al. 1997) and Giardia lamblia (Arkhipova and Morrison 2001). HeT-A, TAHRE, and TART are found at Drosophila telomeres in tandem arrays. HeT-A, the most abundant Drosophila telomeric element, contains a single ORF encoding a Gag-like RNA-binding protein, but lacks reverse transcriptase (RT). It is proposed that the RT necessary for its transposition might be provided in trans, perhaps by TART (Rashkova et al. 2002). TART ORF2 encodes a reverse transcriptase related to the catalytic subunit of telomerase (Levis et al. 1993). The recently discovered TAHRE element shows extensive similarity to $H e T-A$, but contains a second ORF, which encodes a reverse transcriptase (Abad et al. 2004b). A HeT-A promoter located in the $3^{\prime}$ region of the element directs synthesis of a downstream neighbor (Danilevskaya et al. 1997). The TART element was shown to be transcribed bidirectionally using a putative internal sense promoter and antisense one that was localized within the $1-\mathrm{kb}$ region of the TART $3^{\prime}$ end (Danilevskaya et al. 1999). Maintenance of Dro- 
sophila telomere length is mediated by HeT-A and TART transpositions to chromosome ends as well as by terminal recombination/gene conversion (Mikhailovsky et al. 1999; Kahn et al. 2000). Most of the observed spontaneous attachments to telomeres are HeT-A transpositions (Biessmann et al. 1992a; Kahn et al. 2000; Golubovsky et al. 2001), but TART attachments were also detected (Sheen and Levis 1994).

The spn-E and $a u b$ genes, encoding an RNA helicase and a protein of Argonaute family, respectively (Gillespie and Berg 1995; Harris and Macdonald 2001; Kennerdell et al. 2002), were shown to be involved in double-stranded RNA (dsRNA)-triggered RNA interference (RNAi) in embryos (Kennerdell et al. 2002), in transcriptional silencing of transgenes (Pal-Bhadra et al. 2004), and in the control of Drosophila retrotransposon transcript abundance in the germline, especially in ovaries (Aravin et al. 2001; Kogan et al. 2003; Vagin et al. 2004). No effects of RNAi gene mutations on HeT-A and TART expression and telomere structure were observed in somatic tissues (Perrini et al. 2004). Here, we show that increased HeT-A and TART transcript abundance in ovaries, owing to RNAi mutations, is correlated with a high frequency of telomeric element attachments to broken chromosome ends. Addition of HeT-A or TART to a truncated X chromosome, with a break in the upstream regulatory region of yellow, activates yellow expression in aristae, which enables us to monitor the elongation events (Kahn et al. 2000; Savitsky et al. 2002). Using this genetic system, we studied the effects of RNAi mutations on the frequency and molecular nature of telomeric attachments. A high frequency of TART but not HeT-A attachments in heterozygous RNAi mutants suggests that TART may be the primary target of the RNAi-based silencing mecha- nism. These results highlight for the first time the importance of TART, but not the more abundant HeT-A element, in Drosophila telomere maintenance. We found the disappearance of short TART and HeT-A RNAs in spn-E mutant ovaries, strongly suggesting an RNAi-based pathway in the control of telomere maintenance in the Drosophila germline.

\section{Results}

Different patterns of HeT-A and TART expression in ovaries of spn-E and aub mutants

$H e T-A$ transcripts were shown earlier to accumulate in nurse cells and in the growing oocyte in the ovaries of spn- $E^{1}$ and $a u b^{Q C 42}$ mutants (Vagin et al. 2004). In situ RNA hybridization demonstrates the increased TART sense transcripts in cytoplasm of nurse cells, particularly

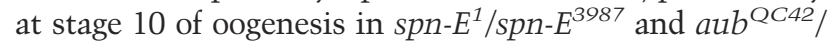
$a u b^{H N}, a u b^{Q C 42} / a u b^{N 11}, a u b^{Q C 42} / a u b^{\text {sting-1 }}, a u b^{Q C 42} /$ $a u b^{\Delta P-3 a}$ heteroallelic flies (Fig. 1A). Ovaries of $s p n-E$ and $a u b$ homozygous mutants contain a small percentage of late-stage egg chambers as a result of strong disturbance of oogenesis (Gillespie and Berg 1995; Wilson et al. 1996). The late stages missing in the mutants led us to the incorrect conclusion that these RNAi mutations exert no effect on TART expression in ovaries (Vagin et al. 2004). The increase of $\mathrm{HeT}$-A transcripts in the nurse cells and oocytes of $a u b^{Q C 42} / a u b^{Q C 42}, a u b^{Q C 42} / a u b^{N 11}$ and $a u b^{Q C 42} / a u b^{\Delta P-3 a}$ individuals occurred in no more than a half of the examined ovaries (Fig. 1B) and sometimes resembled a mosaic $H e T-A$ overexpression in separate ovarioles of the egg chamber. The $a u b^{Q C 42} / a u b^{\text {sting-1 }}$ trans-heterozygotes show no effect on $H e T$ - $A$ transcript

Figure 1. Effect of spn-E and $a u b$ mutations on the abundance of HeT-A and TART transcripts in ovaries observed by in situ RNA hybridization. (A) TART riboprobes detecting sense (s, upper panels) and antisense (as, lower panels) transcripts. TART sense transcripts are detected in the cytoplasm of nurse cells at the late stage of oogenesis in $s p n-E^{1} / s p n-E^{h l s 3987}$ and $a u b^{Q C 42} / a u b^{\Delta P-3 a}$ ovaries. Identical staining was observed in $a u b^{Q C 42} / a u b^{H N}$, aub $b^{Q C 42} / a u b^{N 11}$, and $a u b^{Q C 42} / a u b^{\text {sting-1 }}$ ovaries (data not shown). TART antisense transcript abundance is not affected by spn-E and $a u b$ mutations. (B) Detection of $H e T-A$ sense transcripts in aub ovaries. HeT-A transcripts are detected in the cytoplasm of nurse cells and in the growing oocyte (arrows) in $a u b^{Q C 42} / a u b^{\Delta P-3 a}$ ovaries. The two panels on the right show the mosaic character of HeT-A expression detected only in separate ovarioles. Identical staining was observed in $a u b^{Q C 42} / a u b^{H N}$ and $a u b^{\text {QC42 }} / a u b^{N 11}$ (data not shown). The $a u b^{Q C 42} / a u b^{\text {sting-1 }}$ trans-heterozygotes show

A
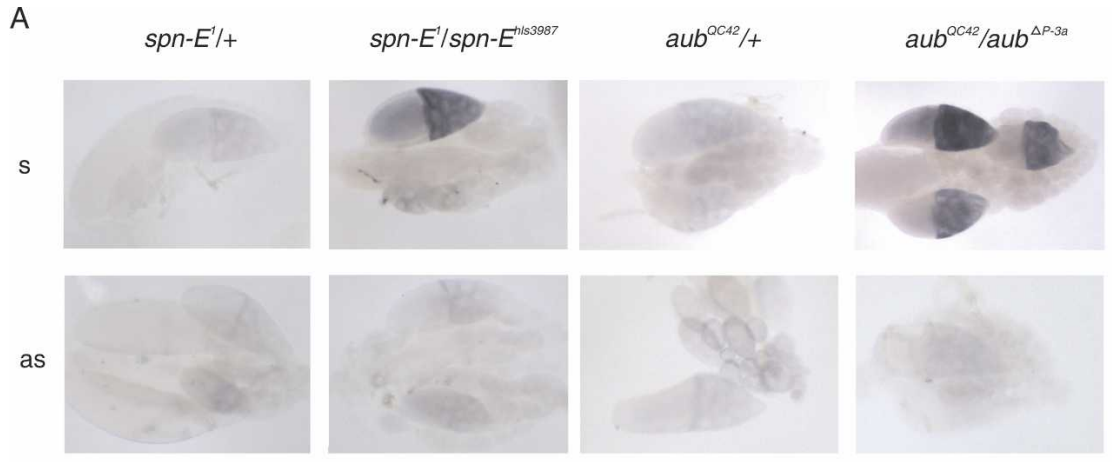

B

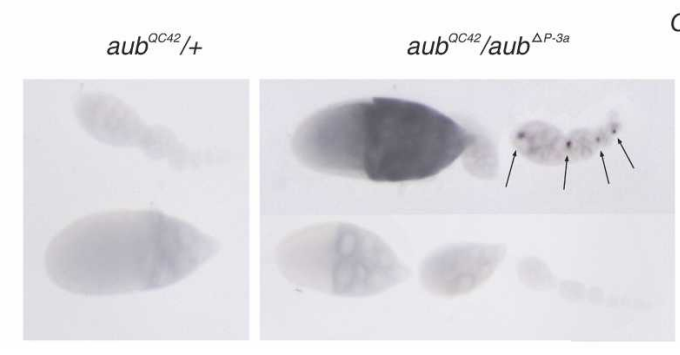

C

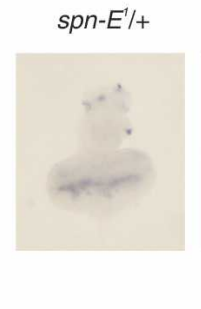

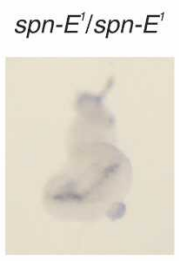

no effect on HeT-A transcript abundance (data not shown). (C) Detection of sense HeT-A transcripts in eye-antennal discs of spn-E larvae. No difference in $H e T-A$ expression in the cells of the morphogenetic furrow of eye-antennal discs of $\operatorname{spn}-E^{1} /+$ and $s p n-E^{1} / s p n-E^{1}$ larvae is observed. Genotypes are indicated at the top of panels. 
abundance (data not shown), whereas TART expression is up-regulated in the nurse cells of the all tested heteroallelic aub mutant flies. In contrast to $H e T-A$, we observed no TART transcript accumulation in the oocytes of RNAi mutants. Antisense TART transcripts are detected in the cytoplasm of nurse cells, but no increase in their abundance was found in RNAi mutants (Fig. 1A). Expression of TART sense and antisense transcripts in the nurse cells suggests the possibility of TART-specific dsRNA formation. HeT-A antisense transcripts are not detected in ovaries of RNAi mutants by in situ RNA hybridization (data not shown) and by Northern analysis in adult flies (Danilevskaya et al. 1999). Thus, an increase of both HeT-A and TART expression in ovaries of the spn-E and $a u b$ mutants was found, while the patterns of their expression are different: HeT-A transcripts are detected both in oocyte and nurse cells, whereas TART transcripts are accumulated substantially in the nurse cells at late stages.

HeT-A was shown to be transcriptionally active in proliferating somatic tissues such as brain and imaginal discs (George and Pardue 2003; Walter and Biessmann 2004). We observed no difference in the expression level of HeT-A in the actively dividing cells of the second mitotic wave in eye-antennal discs of $s p n-E^{1} /+$ and $s p n-E^{1} /$ spn- $E^{1}$ larvae (Fig. 1C). These data highlight a specific role for RNAi genes in the regulation of telomeric retrotransposon expression in the germline.

Increased frequency of telomere attachments in spn-E and aub mutants

To study the frequency of telomeric element attachments to chromosome ends in RNAi mutants, we used the truncated $\mathrm{X}$ chromosomes (designated $\mathrm{y}^{T D}$ ) with a break in the yellow locus that had been used earlier to investigate the effect of HP1 (heterochromatic protein 1) and $\mathrm{Ku}$ proteins on telomere elongation (Savitsky et al. 2002; Melnikova et al. 2005). The break is located in the upstream regulatory region and results in the $\mathrm{y}^{2}$-like phenotype with yellow aristae. Addition of HeT-A or TART retroelements can be monitored by a yellow-toblack change in aristae pigmentation (Savitsky et al. 2002, 2003). The $y^{T D}$ chromosome is lethal as a result of several vital genes deleted in the region distal to the yellow gene. The y ac chromosome used as a homolog in this test has a deletion of the yellow and achaete genes but not of vital genes, and thus allowed us to monitor changes of yellow expression on the $y^{T D}$ chromosome. This system provides an opportunity to estimate the frequency of telomeric element retrotranspositions to the broken chromosome end (Fig. 2A). Moreover, deletion of the yellow locus in the $y$ ac $\mathrm{X}$ chromosome a priori obviates the possibility of terminal elongation using the homologous template to perform gene conversion (Kahn et al. 2000; Savitsky et al. 2002).

The emergence of flies with pigmented aristae was monitored in two lines carrying different $y^{T D}$ chromosomes and spn-E or $a u b$ mutations in a heterozygous state (see Materials and Methods). Two alleles of spn-E,
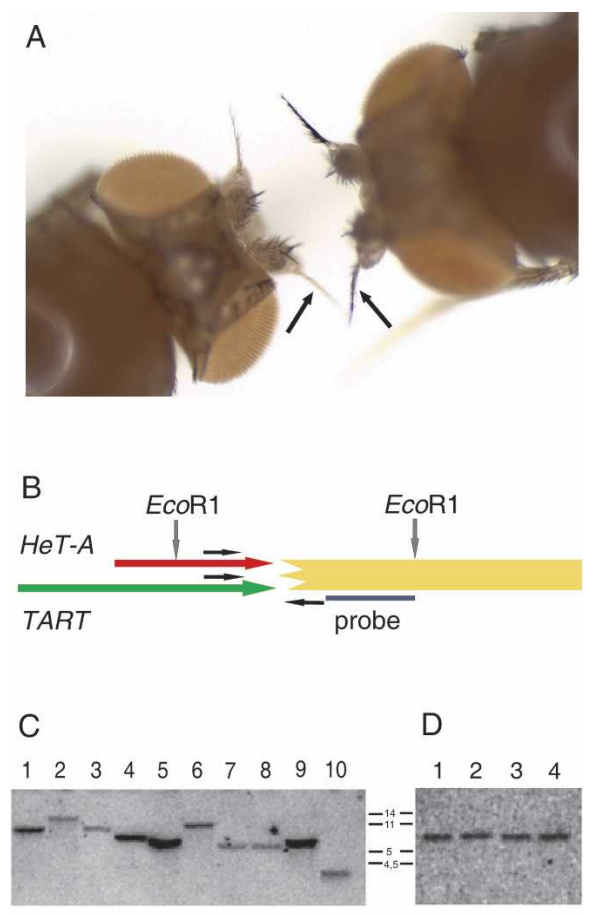

Figure 2. Molecular structure of the HeT-A/TART attachments to the broken chromosome end. (A) Yellow (left)-to-black (right) change in pigmentation of aristae (indicating by arrows) is a result of addition of $H e T-A$ or TART retroelements to the broken X chromosome truncated upstream of the yellow gene. (B) Schematic representation of HeT-A/TART attachments to the broken yellow gene. The yellow region is shown as a yellow box. The horizontal red and green arrows show HeT-A/TART attachments, respectively. Vertical arrows point to EcoRI restriction sites. The solid horizontal line indicates a yellow probe used for Southern analysis. The junctions between yellow and new DNA attachments were amplified using primers schematically indicated as small horizontal arrows. $(C)$ Southern blot analysis of DNAs prepared from the progeny of individual $y^{T D} / y a c$; spn- $E^{1} / M K R S$ flies with yellow aristae (without attachments, lane 10) and black aristae (with TART or HeT-A attachments, lanes 1-6 and 7-9, respectively). DNA was digested by EcoRI enzyme. The filter was hybridized with the yellow probe indicated in $B$. The $4-\mathrm{kb}$ fragment in lane 10 reflects the distance from the EcoRI site in yellow to the truncated end of the broken chromosome. The $5-15-\mathrm{kb}$ bands in lanes 1-6 correspond to the different size attachments of TART elements. The $5.0-\mathrm{kb}$ bands in lanes $7-10$ correspond to attachments of HeT-A elements carrying a conservative EcoRI site in the $3^{\prime}$ region. $(D)$ Southern analysis of DNA isolated from F2 progeny of four females with black aristae (TART attachments), the descendants of an individual $y^{T D} / y a c$; $a u b^{Q C 42} / C y O$ fly with yellow aristae. Hybridized fragments are identical, confirming TART addition at the earlier premeiotic stages of oogenesis. DNA size markers are indicated in kilobases.

spn- $E^{1}$, and spn- $E^{\text {hls3987, as well as aub }}{ }^{Q C 42}$ were used. Table 1 shows the combined results for both $y^{T D}$ chromosomes, indicating an increase of the emergence of flies with black aristae. Homozygous spn-E and aub mutant females are sterile; thus, the observed effect may be 
Table 1. Frequencies of terminal attachments in spn-E and aub mutants

\begin{tabular}{|c|c|c|c|c|c|c|}
\hline \multirow[b]{2}{*}{ RNAi mutations } & \multicolumn{5}{|c|}{ No. of flies displaying a new y phenotype/total no. in generation } & \multirow[b]{2}{*}{$\% \mathrm{Q}\left(\mathrm{F}_{2}-\mathrm{F}_{5}\right)^{2}$} \\
\hline & $\mathrm{F}_{1}$ & $\mathrm{~F}_{2}$ & $\mathrm{~F}_{3}$ & $\mathrm{~F}_{4}$ & $\mathrm{~F}_{5}$ & \\
\hline spn-E $E^{1} / M K R S$ & $1 / 569$ & $3 / 146$ & $41 / 1281$ & $13 / 1162$ & $11 / 759$ & 2.1 \\
\hline spn-E $E^{h l s 3987} / M K R S$ & $1 / 556$ & $36 / 416$ & $18 / 156$ & $35 / 247$ & $37 / 998$ & 6.5 \\
\hline$a u b^{Q C 42} / C y O$ & $0 / 651$ & $3 / 306$ & $10 / 787$ & $17 / 2629$ & - & 0.8 \\
\hline Control $^{\mathrm{b}}$ & $0 / 735$ & $0 / 1005$ & 0/595 & $0 / 744$ & $0 / 824$ & 0.0 \\
\hline
\end{tabular}

${ }^{a} \mathrm{Q}$, average frequency of visible events signifying a new yellow phenotype(s) versus total number of flies scored (as a percentage).

${ }^{\mathrm{b}}$ Combined results of fly score obtained for $y^{T D} / y$ ac; TM6/MKRS and $y^{T D} / y$ ac; CyO/If lines.

attributed to the effect of a single dose of mutant allele. No attachments were detected in control lines. Both spn-E mutations have a strong dominant effect on the frequency of attachments resulting in a 100 times increase in the appearance of flies with black-colored aristae as compared with the earlier observed frequency, $0.04 \%$, of spontaneous telomeric attachments to broken chromosome ends (Kahn et al. 2000). Examination of the aub mutant effect also revealed a considerable, but smaller increase in the frequency of putative terminal attachments. We suggest that both RNAi genes control telomere elongation in Drosophila.

Heterozygous spn-E and aub mutations increase TART attachment to broken chromosome ends

To detect the nature of the attached elements, the junctions between terminal yellow sequences and retrotransposon attachments were analyzed. Genomic DNAs were prepared from the progeny of randomly chosen individual females with restored aristal pigmentation. The junctions between the newly transposed mobile elements and the yellow DNA were determined by DNA amplification with two primers from the yellow gene and the most conserved regions in the 3' part of HeT-A or TART elements (Fig. 2B). Table 2 summarizes the result of PCR analysis. Surprisingly, attachments of HeT-A occurred significantly less frequently than those of TART elements in spn- $E^{1}$ mutants. No HeT-A additions were observed in the lines carrying spn- $E^{\text {hls3987 }}$ and aub ${ }^{Q C 42}$ mutations.

Sequencing of PCR fragments confirmed that, in most cases, the attached elements occurred at different positions of yellow, which is shortened (70-75 base pairs [bp] per generation) as a result of the expected terminal yellow DNA loss (Biessmann and Mason 1988; Levis et al. 1993; Supplementary Fig. 1). The sequences of HeT-A 3' termini (5'-CCAGCAAAGTTA-3') were conserved and terminated by short oligo(A $)_{3-11}$ stretches. TART attachments are characterized by stretches of oligo $(\mathrm{A})_{20-28}$ at their $3^{\prime}$ termini. Most of the TART elements attached to the broken chromosome are attributed to class $\mathrm{A}, \mathrm{B}$, or $\mathrm{C} 1$ (GenBank sequences U02279, U14101, and AY600955, respectively). The presence of oligo(A) tails, conserved 3 ' sequences of attached elements and attachments to different sites of the shortened yellow gene, confirm the retrotransposition mechanism for TART and HeT-A attachments to a broken chromosome end.
Genetic screening of terminal elongation events in the progeny of individual $y^{T D} / y a c$; spn- $E^{h l s 3987} / M K R S$ and $y^{T D} / y a c ; a u b^{Q C 42} / C y O$ females revealed three cases in which several flies with black aristae arose from the same cross. PCR analysis revealed TART attachments in all cases. Sequencing in each of these cases revealed that clusters of flies with black aristae contained identical junctions (Supplementary Fig. 1). Hence, terminal elongation may occur at premeiotic stages of oogenesis.

Southern analysis confirmed elongation of a terminally deleted chromosome (Fig. 2C). Genomic DNA from flies with yellow aristae, $\mathrm{A}^{-}$(in other words, without HeT-A/TART attachments), and black aristae, $\mathrm{A}^{+}$ (with attachments), were digested with EcoRI. A PCR fragment from yellow upstream of the EcoRI site was used as a probe (Fig. 2B). EcoRI recognizes a site in the yellow sequence $\sim 4 \mathrm{~kb}$ proximal to the terminus of the original broken chromosome, producing a fragment corresponding in size to $\mathrm{A}^{-}$genomic DNA. EcoRI is a conserved site in the $3^{\prime}$ region of most HeT-As. No EcoRI recognition sites were detected in most TART sequences. Fragments of $\sim 5.0 \mathrm{~kb}$ encompassing $\sim 4 \mathrm{~kb}$ of terminal yellow sequence and $\sim 1.0 \mathrm{~kb}$ of $\mathrm{HeT}-\mathrm{A}$ are detected by Southerns in $\mathrm{A}^{+}$genomic DNA of the flies in which PCR analysis earlier revealed HeT-A attachments (Fig. 2C, lanes 7-10). Southern analysis reveals fragments of different sizes (from $\sim 5$ to $15 \mathrm{~kb}$ ) when DNA from $\mathrm{A}^{+}$flies with TART additions is digested (Fig. 2C, lanes 1-6). This indicates either attachment of TART elements having polymorphic EcoRI site or addition of truncated TART elements of different sizes, because generation of retroelement copies truncated at the $5^{\prime}$ end is a characteristic feature of RT as a result of its low processivity. This latter observation is consistent

Table 2. Identification of telomeric elements attached to the terminally deleted $X$ chromosome in spn-E and aub mutants

\begin{tabular}{lccc}
\hline \multirow{2}{*}{$\begin{array}{l}\text { Attachment } \\
\text { type }\end{array}$} & \multicolumn{3}{c}{ RNAi mutations } \\
\cline { 2 - 4 } & spn-E & $s p n-E^{\text {hls3987 }}$ & $a u b^{\text {QC42 }}$ \\
\hline TART & 29 & 38 & 16 \\
HeT-A & 6 & - & - \\
Total $^{\text {a }}$ & 35 & 38 & 16 \\
\hline
\end{tabular}

${ }^{a}$ Number of analyzed lines with black-colored aristae. 
with a retrotransposition mechanism of TART attachments to a broken terminus.

Fragments detected in genomic DNA of flies from individual clusters, were of the same size (Fig. 2D), confirming TART addition at the earlier premeiotic stages of oogenesis.

Thus, a dominant effect of the tested RNAi mutations was observed in the increased frequency of TART retrotranspositions to broken chromosome ends. However, we failed to detect an increase in the number of total genomic HeT-A and TART copies in spn- $E^{1} /+$, spn$E^{h 1 s 3987} /+$ and $a u b^{Q C 42} /+$ lines (data not shown). This observation suggests that the telomere length of native chromosomes is not increased significantly. Telomere elongation was detected in Su(var)205 mutants only in those lines that have been maintained for many years (Savitsky et al. 2002), indicating that attachment of retrotransposons to native telomeres is slower than that to a truncated chromosome or that the aristae test is more sensitive than tests for overall telomere length.

HeT-A transposes to chromosome ends in the oocytes of homozygous spn-E mutants

HeT-A expression was shown to be strongly up-regulated in the ovaries of homozygous spn- $E^{1}$ females, resulting in accumulation of $H e T-A$ transcripts at the anterior of the oocyte (Vagin et al. 2004). Since we are unable to estimate frequency of terminal attachments in a progeny of sterile homozygous RNAi mutants, we studied a correlation between $\mathrm{HeT}$ - $\mathrm{A}$ transcript abundance and telomere elongation in ovaries of homozygous spn-E flies carrying a $y^{T D}$ chromosome. Approximately 50 mature oocytes, which are rarely produced in ovaries of homozygous spn-E mutants, were dissected from 10 $s p n-E^{1} / s p n-E^{1}$ flies with yellow aristae, and DNA was isolated. This DNA sample contains oocyte DNA as well as DNA from surrounding follicular cells where no effect of RNAi mutations on HeT-A expression was observed. Genomic DNA was isolated also from carcasses of females after gonad removal. PCR analysis using primers from yellow and the $3^{\prime}$ conservated termini of $\mathrm{HeT}-\mathrm{A} / \mathrm{TART}$, followed by nested PCR was performed. A set of bands was detected in the DNA sample from oocytes when $H e T$-A-specific primers were used. No PCR bands were revealed in DNA from carcasses. Sequencing of PCR products revealed at least five independent $H e T-A$ attachments to truncated chromosome termini (Supplementary Fig. 2A). The same result was obtained when oocytes from a heteroallelic $y^{T D} / y$ ac; spn- $E^{1} / s p n-E^{h l s 3987}$ female were analyzed (Supplementary Fig. 2B). In this case, seven independent HeT-A attachments were observed in the DNA isolated from oocytes of an individual female. Thus, drastic accumulation of $H e T-A$ transcripts in spn- $E^{1 /}$ $s p n-E^{1}$ and $s p n-E^{1} / s p n-E^{h l s 3987}$ oocytes results in a high frequency of $\mathrm{HeT}$ - $\mathrm{A}$ transpositions to the chromosome end, but no TART attachments were found in these cases.

\section{RT-PCR analysis of TART and HeT-A expression in spn-E ovaries}

To elucidate a phenomenon of preferential TART attachments in ovaries of heterozygous spn-E mutants, but much more frequent $H e T-A$ attachments in ovaries of spn-E homozygous flies, we tested for a dosage effect of RNAi mutations on the expression of both retrotransposons. The levels of $H e T-A$ and TART transcripts were compared by RT-PCR using an oligo(dT) primer in ovaries of $y^{T D} / y a c ; T M 6 / M K R S, y^{T D} / y a c ; s p n-E^{1} / M K R S$ and $y^{T D} / y$ ac; spn- $E^{1} / s p n-E^{h 1 s 3987}$ flies. Since the level of antisense TART and HeT-A transcripts detected by in situ RNA hybridization is not affected in RNAi mutants, observed changes may be attributed to the abundance of sense TART and HeT-A transcripts. We observed increased TART transcript abundance in ovaries from heterozygous spn- $E^{1}$ flies and, further, an increase of TART expression in spn- $E^{1} / s p n-E^{h l s 3987}$ ovaries as compared with $y^{T D} / y$ ac; TM6/MKRS ovaries (Fig. 3A). However, while a statistically significant but small dosage effect of spn- $E^{1}$ on HeT-A expression was detected in ovaries of spn- $E^{1} /+$ females, a drastic accumulation of $H e T-A$ transcripts was observed in ovaries of $s p n-E^{1} / s p n-E^{h l s 3987}$ flies (Fig. 3B). Thus, strong derepression of HeT-A in homozygous spn-E mutants provides an explanation for the high frequency of $H e T-A$ attachments to the broken chromosome ends in ovaries from homozygous mutant females. TART may be considered a primary target of RNAi genes owing to the obvious dosage effect of $s p n-E$ and $a u b$ (data not shown) mutations on its expression.

spn-E mutations eliminate short HeT-A and TART RNAs in ovaries

$a u b$ and spn-E are required for artificial RNAi in oocytes and embryos (Kennerdell et al. 2002). Mutations in both
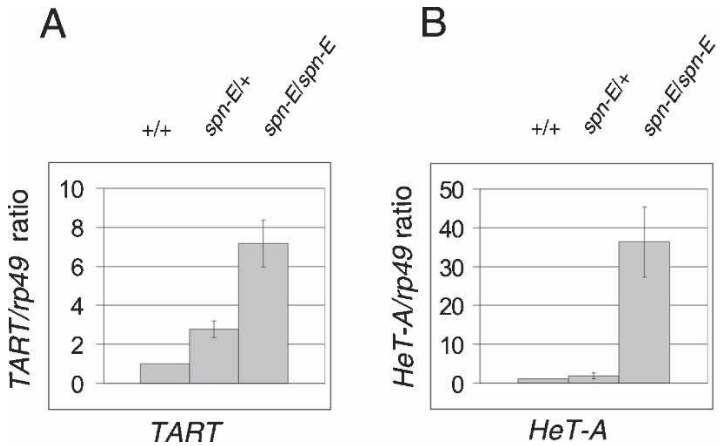

Figure 3. Suppression effect of $s p n-E$ on TART and HeT-A expression. RT-PCR analysis of TART $(A)$ and HeT-A $(B)$ transcript amounts in ovaries of $y^{T D} / y$ ac; spn- $E^{1} / M K R S$ (spn-E/+) and $y^{T D} / y a c$; spn- $E^{1} / s p n-E^{h l s 3987}$ (spn-E/spn-E) flies. Histograms display the quantification of five experiments. Bars represent the ratios of TART or HeT-A to rp49 transcript abundance in $y^{T D} / y$ ac; spn-E $/ M K R S(s p n-E /+)$ and $y^{T D} / y$ ac; spn- $E^{1} / s p n-$ $E^{h 1 s 3987}$ (spn-E/spn-E) ovaries related to this ratio in $y^{T D} / y a c$; $T M 6 / M K R S(+/+)$. Dosage effect of spn-E on TART expression is demonstrated $(A)$, while drastic accumulation of HeT-A transcripts is observed only in ovaries of spn-E/spn-E flies $(B)$. 
genes eliminate the short Suppressor-of-Stellate RNA involved in repression of homologous, testes-expressed Stellate genes by a natural dsRNAi-mediated mechanism (Aravin et al. 2001, 2004). HeT-A- and TARTspecific short RNAs were observed among cloned short RNAs in Drosophila (Aravin et al. 2003). We tested for the presence of short RNA species homologous to TART and $H e T-A$ in the ovary RNA isolated from spn-E mutants and the wild line Gaiano, which is characterized by long telomeric arrays of $H e T-A$ and TART elements (Savitsky et al. 2002; Siriaco et al. 2002). Northern hybridization using TART and HeT-A probes to detect antisense transcripts revealed the presence of heterogeneous 26-29-nt TART and HeT-A RNA species in the ovarian RNA isolated from Gaiano and heterozygous spn-E/+ flies (Fig. 4A,B). The stronger signal for Gaiano is expected, because this line contains longer telomeric arrays. However, no HeT-A or TART short RNAs were detected in RNA from heteroallelic spn- $E^{1} / s p n-E^{h l s 3987}$ ovaries. Expression of microRNA (mir-13b1) in ovaries is not affected by spn-E mutation (Fig. 4A,B), corroborating earlier observations that $s p n-E$ does not affect miRNA production in the germline (Aravin et al. 2004). The signal corresponding to TART short RNA in $s p n-E^{1} /+$ ovaries is faint (Fig. 4A). This observation is consistent with the increased frequency of TART attachments to the broken chromosome ends in $s p n-E^{1} /+$ individuals. A mutation in the not yet identified Tel locus in the Gaiano strain may be responsible for abnormal telomere elongation in this stock (Siriaco et al. 2002). The presence of short HeT-A and TART RNAs in Gaiano indicates that this locus is not related to the production or stabilization

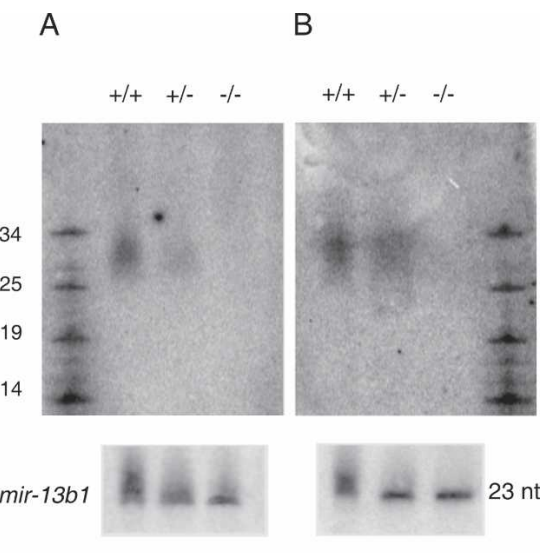

Figure 4. Effect of $s p n-E$ mutation on the presence of the short HeT-A and TART RNAs in ovaries. Northern analysis of RNA, isolated from ovaries of Gaiano $(+/+), s p n-E^{1} /+(+\mid-)$, and spn- $E^{1} / s p n-E^{h l s 3987}$ (-/-) flies. (A) Hybridization with the TART sense riboprobe reveals short TART RNAs of 27-29 nt in size in Gaiano, a faint signal in heterozygous ovaries, and no signal in ovaries of heteroallelic spn-E flies. (B) Hybridization with the $H e T-A$ sense riboprobe. Short HeT-A RNAs are absent in heteroallelic $s p n-E^{1} / s p n-E^{h l s 3987}(-/-)$ flies. The lower panels represent hybridization with an oligonucleotide complementary to the mir-13b1 microRNA. ${ }^{33}$ P-labeled RNA oligonucleotides were used as size markers. of telomeric short RNAs. Our results show a negative correlation between the expression and transposition of telomeric elements and the presence of short RNAs guiding dsRNA-mediated silencing.

\section{Discussion}

An RNAi-based mechanism was proposed to evolve in order to immobilize transposable elements and was found to control expression of endogenous transposable elements and their mobility in different species (Wu-Scharf et al. 2000; Aravin et al. 2001; Sijen and Plasterk 2003; Shi et al. 2004; Svoboda et al. 2004; Kalmykova et al. 2005). Drosophila telomeres are maintained by successive transpositions of specialized telomeric retroelements $H e T-A$ and TART. In this study, we show that transposition of both telomeric elements is under the control of the spn-E and $a u b$ genes, known to be related to the RNAi machinery. Hence, an RNAibased mechanism may be considered not only as a defense against retrotransposon expansion, but also as a regulatory system responsible for proper telomere length maintenance in Drosophila.

$s p n-E$ is required for appropriate localization of mRNA and proteins involved in the establishment of axis formation in the embryo and encodes a member of the DEAD/DE-H protein family possessing RNA-binding and RNA helicase activity (Gillespie and Berg 1995). aub encodes a protein of the Argonaute family that was shown to be a component of the RNAi effector complex RISC (Tomari et al. 2004). aub and spn-E mutations strongly diminished effects of the injected dsRNA into mature oocytes (Kennerdell et al. 2002). Both genes are implicated in small interfering RNA (siRNA)-dependent silencing of testis-expressed Stellate genes (Aravin et al. 2001). Thus, spn-E and $a u b$ are components of RNAibased silencing pathways in Drosophila. Mutations in these genes result in the derepression of a wide spectrum of retrotransposons in the germline, including the HeT-A telomeric element (Aravin et al. 2001; Stapleton et al. 2001; Kogan et al. 2003; Vagin et al. 2004). Here, we demonstrate that spn-E and aub mutations increase the frequency of telomeric element retrotranspositions to broken chromosome termini, suggesting that the RNAi machinery controls telomere length in Drosophila.

Both telomeric elements are shown to be the targets of RNAi. The present results emphasize the differences in the response of HeT-A and TART elements to RNAi mutations. Surprisingly, two different spn-E mutant alleles and an $a u b$ mutation in the heterozygous state increase considerably TART mobility, whereas attachments of HeT-A to broken chromosome ends were detected much more rarely in $s p n-E^{1} /+$ ovaries and are not observed in ovaries of $s p n-E^{\text {hls3987} /+~ a n d ~} a u b^{Q C 42} /+$ flies. One copy of a spn-E mutation is sufficient to increase TART transcript abundance. Strong accumulation of $H e T-A$ transcripts is found only in homozygous mutants, correlating with a high frequency of $H e T-A$ attachments to the broken chromosome ends in the developing oocytes. 
This observation argues that TART is a primary target of the RNAi machinery in ovaries.

TART and HeT-A, in spite of sharing the region of integration, are dissimilar in their structure and expression strategy (Danilevskaya et al. 1999). While both sense and antisense TART transcription was shown, antisense transcripts were more abundant (Danilevskaya et al. 1999). In situ RNA analysis detected sense and antisense TART transcripts in the cytoplasm of nurse cells in the late-stage egg chambers, suggesting a possibility of dsRNA formation. However, we found that the level of antisense TART transcripts is not affected in RNAi mutants. Only sense HeT-A transcription was observed by Northern (Danilevskaya et al. 1999) or by in situ RNA analyses (Walter and Biessmann 2004; our data). Nevertheless, HeT-A- and TART-specific siRNAs were revealed among the cloned short RNA species in Drosophila (Aravin et al. 2003), and we detected short RNAs corresponding to both HeT-A and TART elements by Northern analysis. Antisense HeT-A RNA is probably transcribed at a low level from an unidentified promoter, possibly, from the $H e T-A$ internal region. Actually, a low level of antisense activity of the HeT-A $3^{\prime}$ end was observed (M.-L. Pardue, pers. comm.). While TART transcripts were observed only in the nurse cells, HeT-A transcripts were detected both in the growing oocyte and nurse cells. We propose that TART is a primary target of the RNAi controlling system, since one dose of an RNAi mutation causes preferential TART, but not HeT-A, attachments to broken chromosome ends in ovaries. On the contrary, one dose of a mutant Su(var)205 gene considerably increased the frequency of $H e T-A$ rather than TART attachments to the chromosome ends (Savitsky et al. 2002). Thus, we observed a specific effect of RNAi components on telomeric element expression. Although TART copies are much less abundant in the genome than HeT-A and no TART elements are detected in some telomeres (Levis et al. 1993; Abad et al. 2004a), TART is a conserved component of telomeres in distant Drosophila species (Casacuberta and Pardue 2002, 2003). TART was considered as a source of RT production, thus ensuring retrotranspositions of both TART and HeT-A elements (Rashkova et al. 2002). One may propose that TART supplies an RNAi-regulated template for RT production, thus providing telomere-specific transpositions of both elements.

Drosophila telomeres contain a multisubunit protein complex forming a chromosome cap protecting chromosomes from DNA repair and end-to-end fusions (Cenci et al. 2005). However, no HeT-A or TART sequences were detected at the stably maintained broken chromosome end that is protected from telomere fusions (Biessmann et al. 1990; Mason and Biessmann 1995). Thus, a sequence-independent system performs telomere capping functions. The capping complex contains HP1 (Fanti et al. 1998), HOAP (HP1/ORC associated protein) (Cenci et al. 2003), as well as ATM-kinase and DNA repair MRN complex and the Ku70/Ku80 heterodimer (Bi et al. 2004; Ciapponi et al. 2004; Oikemus et al. 2004; Silva et al. 2004; Song et al. 2004; Melnikova et al. 2005). HP1 and the $\mathrm{Ku}$ heterodimer act also as negative regulators of telomere elongation by retrotransposition of telomeric elements (Savitsky et al. 2002; Melnikova et al. 2005). Deficiencies that remove either the Ku70 or the Kus0 gene increase the transposition rate of $H e T-A$ and TART elements but exert no effect on the HeT-A expression, suggesting that $\mathrm{Ku}$ proteins control the accessibility of the telomere to transposition events (Melnikova et al. 2005). At the same time, mutations in the Su(var)205 gene increase both transcript abundance of $\mathrm{HeT}$-A and $T A R T$ and the frequency of their attachments to chromosome ends (Savitsky et al. 2002; Perrini et al. 2004). RNAi affects both telomeric retrotransposon expression and the rate of transposition to the telomere. Probably, this effect is mediated through HP1 recruitment and silencing of $H e T-A$ and/or TART chromatin.

siRNAs produced from telomeric elements TART and $H e T$ - $A$ belong to the long size class (25-29 nt) in contrast to 21-22-nt RNAs guiding post-transcriptional RNAi (Elbashir et al. 2001). In plants, long siRNAs are associated with RNA-directed DNA methylation and play an essential role in the transcriptional retrotransposon silencing (Hamilton et al. 2002). dsRNA and proteins of the RNAi machinery can direct chromatin alteration to homologous DNA sequences and induce transcriptional silencing (Matzke and Birchler 2005). RNAi mutations cause delocalization of HP1 in yeast and Drosophila (Hall et al. 2002; Volpe et al. 2002; Pal-Bhadra et al. 2004). Actually, the increase in accessibility of HeT-A chromatin and its enrichment in K9-acetylated H3 histone were revealed in ovaries of spn-E mutants (M. Klenov and S. Lavrov, unpubl.). It is also possible that TART and/or HeT-A short RNAs can be targeted to telomeric repeats in a transcriptional silencing complex.

RNAi disruption affects neither HeT-A and TART expression, nor telomere fusions in somatic cells (Perrini et al. 2004). We failed to observe the effect of $s p n-E$ mutations on HeT-A expression, even in actively dividing cells of imaginal discs, where HeT-A expression was found (George and Pardue 2003; Walter and Biessmann 2004). Our data indicate a crucial role of the RNAi machinery in the regulation of telomere elongation in germinal cells. The appearance of a cluster of individuals with identical retroelement attachments indicates that dsRNA-mediated control of terminal elongation may occur at premeiotic stages of oogenesis.

We have demonstrated for the first time that expression and retrotransposition of specific telomeric repeats is under control of an RNAi-based system in the Drosophila germline. In this case, the telomerase-dependent mechanism of telomere stability is substituted by retrotranspositions. Interestingly, telomerase-dependent telomere functioning during meiosis in the yeasts Schizosaccharomyces pombe and Tetrahymena is also under the control of RNAi machinery (Hall et al. 2003; Mochizuki and Gorovsky 2005). These observations and our data indicate that dsRNA-mediated regulation of telomere dynamics in the germline may be a general phenomenon independent of a mode of telomere maintenance. 


\section{Materials and methods}

Drosophila strains and genetic crosses

The strains bearing spindle-E (spn-E) mutations were $r u^{1} s t^{1} s p n-E^{1} e^{1}$ $c a^{1} / \mathrm{TM} 3, S b^{1} e^{s}$ (bearing a point mutation in the helicase domain), and $r u^{1} s t^{1} s p n-E^{h 1 s 3987} e^{1} c a^{1} / \mathrm{TM} 3, S b^{1} e^{s}$ (caused by $P$-element insertion). The $a u b$ mutants were $a u b^{Q C 42} / C y O$, $a u b^{H N} / C y O, a u b^{N 11} / C y O$, aub $b^{\text {sting1 }} / C y O$, and $a u b^{\Delta P 3 a} / C y O$.

To study the frequency of HeT-A/TART attachments to broken chromosome ends, we used a terminally truncated chromosome with a break in the yellow locus designated $y^{T D}$. The $y^{T D}$ chromosome is lethal. The homologous y ac X chromosome has a deletion of yellow and achaete loci but not of any vital genes. Two lines, $y^{T D 4} / y a c$ and $y^{T D 5} / y a c$, carrying deficiencies terminating in the region $\sim 1.3-1.5 \mathrm{~kb}$ upstream of the yellow transcription start site, were used. The $\mathrm{y}^{T D} / \mathrm{y} a c$; TM6/MKRS females were crossed to y $a c$; $s p n-E^{1} / M K R S$ males. In the progeny, $y^{T D} / y a c ; s p n-E^{1} / M K R S$ females were crossed to y ac; spn- $E^{1} /$ $M K R S$ males for five successive generations to determine the appearance of flies with black aristae. The same crossing was used to study the spn- $E^{h l s 3987}$ allele. The $y^{T D} / y$ ac; TM6/MKRS line was used as a control. $y^{T D} / y$ ac; $C y O / I f$ females were crossed to y ac; $a u b^{Q C 42} / C y O$ males to obtain $y^{T D} / y a c$; $a u b^{\mathrm{QC} 42} / \mathrm{CyO}$ females. The $y^{T D} / \mathrm{y} a c ; C y O / I f$ line was used as a control.

\section{Northern and in situ RNA analyses}

Northern analysis of short RNAs was done according to Aravin et al. (2001). ${ }^{32} \mathrm{P}$-labeled riboprobes corresponding to the sense strands of TART and HeT-A were synthesized. In situ RNA analysis was carried out according to the earlier described procedure (Kogan et al. 2003) using DIG-labeled strand-specific TART and HeT-A riboprobes. Plasmids containing the cloned PCR fragments of retrotransposons were used as transcription templates. TART: A PCR-amplified fragment using primers 5 'TTCTATCAACAGGCTGTCCACAGGTT-3' and 5'-CCTTCG TAGTCGGGTAGGATTATTCGT-3' corresponding to GenBank sequence DMU02279 was cloned into pBS SK${ }^{-}$. HeT-A: A PCRamplified fragment using primers $5^{\prime}$-TCATTGACGATAC

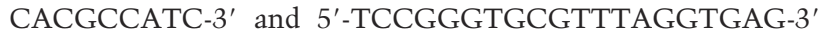
corresponding to GenBank sequence DMU06920 was cloned into $\mathrm{pBS} \mathrm{SK}^{-}$. Hybridization with ${ }^{32} \mathrm{P}$-end-labeled oligonucleotide 5'-ACTCGTCAAAATGGCTGTGATA-3' complementary to mir13b1 was used as a loading control.

\section{PCR and RT-PCR analyses}

DNA samples were isolated from the $\mathrm{F} 2$ progeny of individual $y^{T D} / y$ ac; spn- $E^{1} / M K R S, y^{T D} / y$ ac; spn- $E^{h l s 3987} / M K R S$ or $y^{T D} / y$ ac; $a u b^{Q C 42} / C y O$ females with black aristae according to the standard method (Ashburner 1989). PCR was done using different combinations of primers corresponding to TART/HeT-A and yellow sequences as follows: TART-specific primers: TART1, 5'-CGAAACGCAACAACAAAATGG-3'; TART2, 5'-AAGGA TGGGCAACAGTGCTATC-3'; TART3, 5' ${ }^{\prime}$ TGGACGGCCAG AACGTGAACCA-3'; HeT-A-specific primers: A2H, 5'-TTGC AATATGTTAATGTTACCAGTCCATG-3'; M1, 5'-CTGTCT CCGTACCTCCACCAGC-3'; M3, 5'-CCTATATATTGCACA TACTGTAATCAAAG-3'; yellow-specific primers corresponding to GenBank sequences X06481 and X04427: y13, 5'-AAT ATTTTGTTTCCGCTAGTTATTG-3'; y12, 5'-ATTGGATTT CGATTGGGCGTCAC-3'; y5, 5' -CAGGAGGCTCGTGCATA GAATGC-3'; y9, 5'-GGTTCAGTGTTCGGGTAATCAGGTG-3';

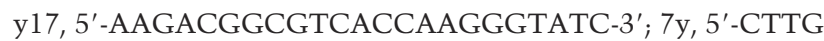
CGGCGATGGTCATTAGAGC-3'. PCR fragments were sequenced directly or cloned in pTR19R.

To reveal TART/HeT-A attachments in oocytes of sterile spn- $E$ mutants, genomic DNA was isolated from oocytes of $y^{T D} / y a c ; s p n-E^{1} / s p n-E^{1}$ and $y^{T D} / y a c ; s p n-E^{1} / s p n-E^{h l s 3987}$ females with yellow-colored aristae and carcasses of flies after gonad removal. PCR with primers y12 or y17 and A2H/TART1 following by nested PCR with y13 or y9 and M1/TART2 was performed using $y^{T D} / y a c$; spn- $E^{1} / s p n-E^{1}$ or $y^{T D} / y a c$; $s p n-E^{1} /$ $s p n-E^{h l s 3987}$ DNAs, respectively. PCR products were cloned and sequenced.

RT-PCR was done according to described procedure (Aravin et al. 2001) using pairs of primers corresponding to HeT-A and TART as indicated in the previous section of Materials and Methods, and with rp49 (5'-ATGACCATCCGCCCAGCAT AC-3' and 5'-CTGCATGAGCAGGACCTCCAG-3') as a loading control. cDNA was synthesized using an oligo(dT) primer. The results of RT-PCR analyses were evaluated using the program BioDocAnalyze1.0.

Southern analysis was performed according to the standard method (Ashburner 1989) using as a probe a ${ }^{32} \mathrm{P}$-labeled fragment of yellow obtained by primers $7 \mathrm{y}$ and $\mathrm{y} 9$.

\section{Acknowledgments}

We thank M.-L. Pardue and J. Mason for critical reading of this manuscript and A. Aravin and V. Vagin for helpful methodological advice. This work was supported by the RAS program for Molecular and Cell Biology to V.G. and A.K.; an International Research Scholar award from the Howard Hughes Medical Institute to P.G.; a grant from the Russian Foundation for Basic Researches (06-04-48493), the Russian Foundation for Science School (N 2074, 2003.4), a stipend from the Center for Medical Studies, University of Oslo to M.S.; and a President of the Russian Federation Grant to M.S. (MK-2171.2005.4).

\section{References}

Abad, J.P., de Pablos, B., Osoegawa, K., de Jong, P.J., MartinGallardo, A., and Villasante, A. 2004a. Genomic analysis of Drosophila melanogaster telomeres: Full-length copies of HeT-A and TART elements at telomeres. Mol. Biol. Evol. 21: 1613-1619.

- 2004b. TAHRE, a novel telomeric retrotransposon from Drosophila melanogaster, reveals the origin of Drosophila telomeres. Mol. Biol. Evol. 21: 1620-1624.

Aravin, A.A., Naumova, N.M., Tulin, A.V., Vagin, V.V., Rozovsky, Y.M., and Gvozdev, V.A. 2001. Double-stranded RNA-mediated silencing of genomic tandem repeats and transposable elements in the $D$. melanogaster germline. Curr. Biol. 11: 1017-1027.

Aravin, A.A., Lagos-Quintana, M., Yalcin, A., Zavolan, M., Marks, D., Snyder, B., Gaasterland, T., Meyer, J., and Tuschl, T. 2003. The small RNA profile during Drosophila melanogaster development. Dev. Cell 5: 337-350.

Aravin, A.A., Klenov, M.S., Vagin, V.V., Bantignies, F., Cavalli, G., and Gvozdev, V.A. 2004. Dissection of a natural RNA silencing process in the Drosophila melanogaster germ line. Mol. Cell. Biol. 24: 6742-6750.

Arkhipova, I.R. and Morrison, H.G. 2001. Three retrotransposon families in the genome of Giardia lamblia: Two telomeric, one dead. Proc. Natl. Acad. Sci. 98: 14497-14502.

Ashburner, M. 1989. Drosophila: A laboratory manual. Cold 
Spring Harbor Laboratory Press, Cold Spring Harbor, NY.

Bi, X., Wei, S.C., and Rong, Y.S. 2004. Telomere protection without a telomerase; the role of ATM and Mre11 in Drosophila telomere maintenance. Curr. Biol. 14: 1348-1353.

Biessmann, H. and Mason, J.M. 1988. Progressive loss of DNA sequences from terminal chromosome deficiencies in Drosophila melanogaster. EMBO J. 7: 1081-1086.

Biessmann, H., Carter, S.B., and Mason, J.M. 1990. Chromosome ends in Drosophila without telomeric DNA sequences. Proc. Natl. Acad. Sci. 87: 1758-1761.

Biessmann, H., Champion, L.E., O'Hair, M., Ikenaga, K., Kasravi, B., and Mason, J.M. 1992a. Frequent transpositions of Drosophila melanogaster HeT-A transposable elements to receding chromosome ends. EMBO J. 11: 4459-4469.

Biessmann, H., Valgeirsdottir, K., Lofsky, A., Chin, C., Ginther, B., Levis, R.W., and Pardue, M.L. 1992b. HeT-A, a transposable element specifically involved in "healing" broken chromosome ends in Drosophila melanogaster. Mol. Cell. Biol. 12: 3910-3918.

Bryan, T.M. and Cech, T.R. 1999. Telomerase and the maintenance of chromosome ends. Curr. Opin. Cell Biol. 11: 318324.

Casacuberta, E. and Pardue, M.L. 2002. Coevolution of the telomeric retrotransposons across Drosophila species. Genetics 161: 1113-1124.

2003. Transposon telomeres are widely distributed in the Drosophila genus: TART elements in the virilis group. Proc. Nat1. Acad. Sci. 100: 3363-3368.

Cenci, G., Siriaco, G., Raffa, G.D., Kellum, R., and Gatti, M. 2003. The Drosophila HOAP protein is required for telomere capping. Nat. Cell Biol. 5: 82-84.

Cenci, G., Ciapponi, L., and Gatti, M. 2005. The mechanism of telomere protection: A comparison between Drosophila and humans. Chromosoma 114: 135-145.

Chan, S.W. and Blackburn, E.H. 2002. New ways not to make ends meet: Telomerase, DNA damage proteins and heterochromatin. Oncogene 21: 553-563.

Ciapponi, L., Cenci, G., Ducau, J., Flores, C., Johnson-Schlitz, D., Gorski, M.M., Engels, W.R., and Gatti, M. 2004. The Drosophila Mre11/Rad50 complex is required to prevent both telomeric fusion and chromosome breakage. Curr. Biol. 14: 1360-1366.

Danilevskaya, O.N., Arkhipova, I.R., Traverse, K.L., and Pardue, M.L. 1997. Promoting in tandem: The promoter for telomere transposon HeT-A and implications for the evolution of retroviral LTRs. Cell 88: 647-655.

Danilevskaya, O.N., Traverse, K.L., Hogan, N.C., DeBaryshe, P.G., and Pardue, M.L. 1999. The two Drosophila telomeric transposable elements have very different patterns of transcription. Mol. Cell. Biol. 19: 873-881.

Elbashir, S.M., Lendeckel, W., and Tuschl, T. 2001. RNA interference is mediated by 21 - and 22-nucleotide RNAs. Genes \& Dev. 15: 188-200.

Fanti, L., Giovinazzo, G., Berloco, M., and Pimpinelli, S. 1998. The heterochromatin protein 1 prevents telomere fusions in Drosophila. Mol. Cell 2: 527-538.

George, J.A. and Pardue, M.-L. 2003. The promoter of the heterochromatic Drosophila telomeric retrotransposon, HeT-A, is active when moved into euchromatic locations. Genetics 163: 625-635.

Gillespie, D.E. and Berg, C.A. 1995. Homeless is required for RNA localization in Drosophila oogenesis and encodes a new member of the DE-H family of RNA-dependent ATPases. Genes \& Dev. 9: 2495-2508.

Golubovsky, M.D., Konev, A.Y., Walter, M.F., Biessmann, H., and Mason, J.M. 2001. Terminal retrotransposons activate a subtelomeric white transgene at the $2 \mathrm{~L}$ telomere in Drosophila. Genetics 158: 1111-1123.

Greider, C.W. and Blackburn, E.H. 1985. Identification of a specific telomere terminal transferase activity in Tetrahymena extracts. Cell 43: 405-413.

Hall, I.M., Shankaranarayana, G.D., Noma, K., Ayoub, N., Cohen, A., and Grewal, S.I. 2002. Establishment and maintenance of a heterochromatin domain. Science 297: 22322237.

Hall, I.M., Noma, K., and Grewal, S.I. 2003. RNA interference machinery regulates chromosome dynamics during mitosis and meiosis in fission yeast. Proc. Natl. Acad. Sci. 100: 193198.

Hamilton, A., Voinnet, O., Chappell, L., and Baulcombe, D. 2002. Two classes of short interfering RNA in RNA silencing. EMBO J. 21: 4671-4679.

Harris, A.N. and Macdonald, P.M. 2001. Aubergine encodes a Drosophila polar granule component required for pole cell formation and related to eIF2C. Development 128: 28232832.

Higashiyama, T., Noutoshi, Y., Fujie, M., and Yamada, T. 1997. Zepp, a LINE-like retrotransposon accumulated in the Chlorella telomeric region. EMBO J. 16: 3715-3723.

Kahn, T., Savitsky, M., and Georgiev, P. 2000. Attachment of HeT-A sequences to chromosomal termini in Drosophila melanogaster may occur by different mechanisms. Mol. Cell. Biol. 20: 7634-7642.

Kalmykova, A.I., Klenov, M.S., and Gvozdev, V.A. 2005. Argonaute protein PIWI controls mobilization of retrotransposons in the Drosophila male germline. Nucleic Acids Res. 33: 2052-2059.

Kennerdell, J.R., Yamaguchi, S., and Carthew, R.W. 2002. RNAi is activated during Drosophila oocyte maturation in a manner dependent on aubergine and spindle-E. Genes \& Dev. 16: 1884-1889.

Kogan, G.L., Tulin, A.V., Aravin, A.A., Abramov, Y.A., Kalmykova, A.I., Maisonhaute, C., and Gvozdev, V.A. 2003. The GATE retrotransposon in Drosophila melanogaster: Mobility in heterochromatin and aspects of its expression in germline tissues. Mol. Genet. Genomics 269: 234-242.

Levis, R.W., Ganesan, R., Houtchens, K., Tolar, L.A., and Sheen, F.M. 1993. Transposons in place of telomeric repeats at a Drosophila telomere. Cell 75: 1083-1093.

Mason, J.M. and Biessmann, H. 1995. The unusual telomeres of Drosophila. Trends Genet. 11: 58-62.

Matzke, M.A. and Birchler, J.A. 2005. RNAi-mediated pathways in the nucleus. Nat. Rev. Genet. 6: 24-35.

Melnikova, L., Biessmann, H., and Georgiev, P. 2005. The Ku protein complex is involved in length regulation of Drosophila telomeres. Genetics 170: 221-235.

Mikhailovsky, S., Belenkaya, T., and Georgiev, P. 1999. Broken chromosomal ends can be elongated by conversion in Drosophila melanogaster. Chromosoma 108: 114-120.

Mochizuki, K. and Gorovsky, M.A. 2005. A Dicer-like protein in Tetrahymena has distinct functions in genome rearrangement, chromosome segregation, and meiotic prophase. Genes \& Dev. 19: 77-89.

Oikemus, S.R., McGinnis, N., Queiroz-Machado, J., Tukachinsky, H., Takada, S., Sunkel, C.E., and Brodsky, M.H. 2004. Drosophila atm/telomere fusion is required for telomeric localization of HP1 and telomere position effect. Genes \& Dev. 18: $1850-1861$.

Okazaki, S., Ishikawa, H., and Fujiwara, H. 1995. Structural analysis of TRAS1, a novel family of telomeric repeat-associated retrotransposons in the silkworm, Bombyx mori. Mol. Cell. Biol. 15: 4545-4552. 
Pal-Bhadra, M., Leibovitch, B.A., Gandhi, S.G., Rao, M., Bhadra, U., Birchler, J.A., and Elgin, S.C. 2004. Heterochromatic silencing and HP1 localization in Drosophila are dependent on the RNAi machinery. Science 303: 669-672.

Pardue, M.L. and DeBaryshe, P.G. 2003. Retrotransposons provide an evolutionary robust non-telomerase mechanism to maintain telomeres. Annu. Rev. Genet. 37: 485-511.

Perrini, B., Piacentini, L., Fanti, L., Altieri, F., Chichiarelli, S., Berloco, M., Turano, C., Ferraro, A., and Pimpinelli, S. 2004. HP1 controls telomere capping, telomere elongation, and telomere silencing by two different mechanisms in Drosophila. Mol. Cell 15: 467-476.

Rashkova, S., Karam, S.E., Kellum, R., and Pardue, M.L. 2002. Gag proteins of the two Drosophila telomeric retrotransposons are targeted to chromosome ends. J. Cell Biol. 159: 397-402.

Savitsky, M., Kravchuk, O., Melnikova, L., and Georgiev, P. 2002. Heterochromatin protein 1 is involved in control of telomere elongation in Drosophila melanogaster. Mol. Cell. Biol. 22: 3204-3218.

Savitsky, M., Kahn, T., Pomerantseva, E., and Georgiev, P. 2003. Transvection at the end of the truncated chromosome in Drosophila melanogaster. Genetics 163: 1375-1387.

Sheen, F.M. and Levis, R.W. 1994. Transposition of the LINElike retrotransposon TART to Drosophila chromosome termini. Proc. Nat1. Acad. Sci. 91: 12510-12514.

Shi, H., Djikeng, A., Tschudi, C., and Ullu, E. 2004. Argonaute protein in the early divergent eukaryote Trypanosoma brucei: Control of small interfering RNA accumulation and retroposon transcript abundance. Mol. Cell. Biol. 24: 420-427.

Sijen, T. and Plasterk, R.H. 2003. Transposon silencing in the Caenorhabditis elegans germ line by natural RNAi. Nature 426: 310-314.

Silva, E., Tiong, S., Pedersen, M., Homola, E., Royou, A., Fasulo, B., Siriaco, G., and Campbell, S.D. 2004. ATM is required for telomere maintenance and chromosome stability during Drosophila development. Curr. Biol. 14: 1341-1347.

Siriaco, G.M., Cenci, G., Haoudi, A., Champion, L.E., Zhou, C., Gatti, M., and Mason, J.M. 2002. Telomere elongation (Tel), a new mutation in Drosophila melanogaster that produces long telomeres. Genetics 160: 235-245.

Song, Y.H., Mirey, G., Betson, M., Haber, D.A., and Settleman, J. 2004. The Drosophila ATM ortholog, dATM, mediates the response to ionizing radiation and to spontaneous DNA damage during development. Curr. Biol. 14: 1354-1359.

Stapleton, W., Das, S., and McKee, B.D. 2001. A role of the Drosophila homeless gene in repression of Stellate in male meiosis. Chromosoma 110: 228-240.

Svoboda, P., Stein, P., Anger, M., Bernstein, E., Hannon, G.J., and Schultz, R.M. 2004. RNAi and expression of retrotransposons MuERV-L and IAP in preimplantation mouse embryos. Dev. Biol. 269: 276-285.

Takahashi, H., Okazaki, S., and Fujiwara, H. 1997. A new family of site-specific retrotransposons, SART1, is inserted into telomeric repeats of the silkworm, Bombyx mori. Nucleic Acids Res. 25: 1578-1584.

Tomari, Y., Du, T., Haley, B., Schwarz, D.S., Bennett, R., Cook, H.A., Koppetsch, B.S., Theurkauf, W.E., and Zamore, P.D. 2004. RISC assembly defects in the Drosophila RNAi mutant armitage. Cell 116: 831-841.

Vagin, V.V., Klenov, M.S., Kalmykova, A.I., Stolyarenko, A.D., Kotelnikov, R.N., and Gvozdev, V.A. 2004. The RNA interference proteins and vasa locus are involved in the silencing of retrotransposons in the female germline of Drosophila melanogaster. RNA Biol. 1: 54-58.

Volpe, T.A., Kidner, C., Hall, I.M., Teng, G., Grewal, S.I., and
Martienssen, R.A. 2002. Regulation of heterochromatic silencing and histone H3 lysine-9 methylation by RNAi. Science 297: 1833-1837.

Walter, M.F. and Biessmann, H. 2004. Expression of the telomeric retrotransposon HeT-A in Drosophila melanogaster is correlated with cell proliferation. Dev. Genes Evol. 214: 211-219.

Wilson, J.E., Connell, J.E., and Macdonald, P.M. 1996. aubergine enhances oskar translation in the Drosophila ovary. Development 122: 1631-1639.

Wu-Scharf, D., Jeong, B., Zhang, C., and Cerutti, H. 2000. Transgene and transposon silencing in Chlamydomonas reinhardtii by a DEAH-box RNA helicase. Science 290: 11591162 . 


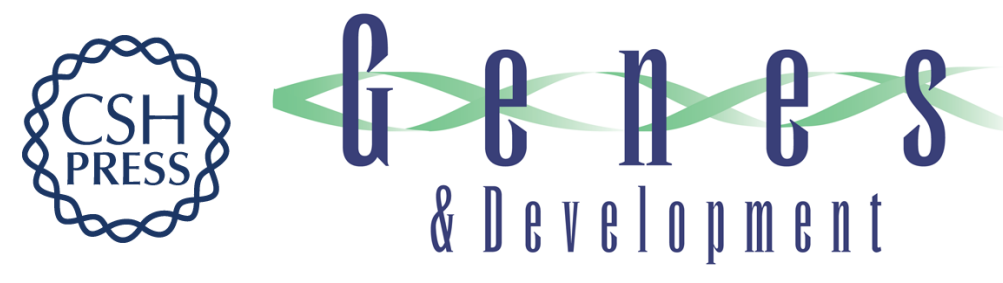

\section{Telomere elongation is under the control of the RNAi-based mechanism in the Drosophila germline}

Mikhail Savitsky, Dmitry Kwon, Pavel Georgiev, et al.

Genes Dev. 2006, 20:

Access the most recent version at doi:10.1101/gad.370206

Supplemental
Material http://genesdev.cshlp.org/content/suppl/2006/01/18/20.3.345.DC1

References This article cites 64 articles, 31 of which can be accessed free at: http://genesdev.cshlp.org/content/20/3/345.full.html\#ref-list-1

License

Email Alerting Receive free email alerts when new articles cite this article - sign up in the box at the top Service right corner of the article or click here.

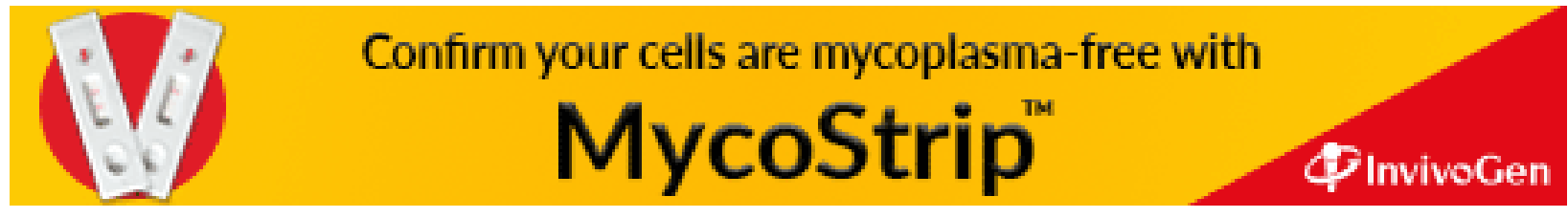

